\title{
Modulation of retrieval processing reflects accuracy of emotional source memory
}

\author{
Adam P.R. Smith, ${ }^{1,2,4}$ Richard N.A. Henson,, ${ }^{1,2}$ Michael D. Rugg, ${ }^{3}$ and Raymond J. Dolan ${ }^{1}$ \\ ${ }^{1}$ Wellcome Department of Imaging Neuroscience, Institute of Neurology, University College London, London WC1E 6BT, \\ United Kingdom; ${ }^{2}$ Institute of Cognitive Neuroscience, University College London, London WC1N 3AR, United Kingdom; \\ ${ }^{3}$ Center for the Neurobiology of Learning and Memory, University of California, Irvine, California 92697-3800, USA
}

\begin{abstract}
There is considerable evidence that encoding and consolidation of memory are modulated by emotion, but the retrieval of emotional memories is not well characterized. Here we manipulated the emotional context with which affectively neutral stimuli were associated during encoding, allowing us to examine neural activity associated with retrieval of emotional memories without confounding the emotional attributes of cue items and the retrieved context. Using a source memory procedure we were also able to compare how retrieval processing was modulated when the emotional encoding context was recollected or not. An interaction between emotional encoding context and accuracy of source memory revealed that successful retrieval of emotional context was associated with activity in left amygdala, and a left frontotemporal network including anterior insula, prefrontal cortex and cingulate. In contrast, when contextual retrieval was unsuccessful, items encoded in emotional contexts elicited enhanced activity in right amygdala and a right-lateralized network that included extrastriate visual areas. These findings indicate distinct effects of emotion on successful and unsuccessful retrieval of source information, including lateralization of amygdala responses.
\end{abstract}

Interactions between emotion and memory are fundamental in the formation and modification of cognitive representations of the value of environmental elements. Several neuroimaging experiments have investigated emotional memory (for review, see Hamann 2001), but few have addressed such interactions at retrieval. One approach to investigating retrieval of emotional information involves examining neural activity associated with recognition of stimuli studied in association with emotional and non-emotional contexts (Maratos and Rugg 2001; Maratos et al. 2001; Smith et al. 2004a,b). The use of test items without intrinsic emotional value avoids confounding the emotional attributes of recognition cues with attributes of retrieved memories, which precludes unequivocal dissociation of mnemonic and online processing effects. We used a recently developed procedure (Maratos and Rugg 2001; Smith et al. 2004a) to identify neural activity associated with explicit retrieval of task-relevant emotional information, and to examine how this was modified by the accuracy of such retrieval.

Previous fMRI studies have reported differences in neural activity elicited by recognition of neutral words studied in the context of emotional versus neutral sentences (Maratos et al. 2001), pictures studied with emotional versus neutral backgrounds (Smith et al. 2004b), and with emotional versus neutral odors (Gottfried et al. 2004). Some of the reported effects were evident in areas supporting retrieval processing, such as dorsolateral prefrontal cortex (DLPFC), whereas others occurred in emotion-sensitive regions such as the amygdala, insula, orbitofrontal cortex (OFC), and ventromedial prefrontal cortex (VMPFC). These findings imply that memory for items with an emotional study history elicits activity in structures associated with processing of emotional stimuli, in addition to modulating those typically engaged during memory retrieval. However, such

\footnotetext{
${ }^{4}$ Corresponding author.

E-mail adam.smith@ucl.ac.uk; fax 442078131420.

Article and publication are at http://www.learnmem.org/cgi/doi/10.1101/ Im.84305.
}

effects may be accounted for by two alternative mechanisms. First, stimuli presented in emotional contexts may, by virtue of association, themselves acquire emotional value and subsequently elicit activity in emotion-processing structures without the necessity to retrieve information about the context. Secondly, recognition of the item may be associated with recollection of the encoding episode, including the emotional context, which subsequently engages emotional processing.

Event-related potentials (ERPs) elicited by recognition of items from emotional versus neutral contexts show two distinct emotion effects (Smith et al. 2004a); one effect onsets prior to the "left parietal old/new effect" (an ERP correlate of episodic retrieval; see Rugg and Allan 2000) whereas the second effect onsets subsequent to the parietal effect. It was suggested that the earlier effect reflected activity elicited by the test item due to a change in its emotional value following the encoding manipulation, whereas the later effect reflected processing of the retrieved context. The scalp topography of late-onsetting emotion effects differed according to whether subjects could make accurate source judgements (Smith et al. 2004a; A.P.R. Smith, R.J. Dolan, and M.D. Rugg, unpubl.). This indicated that qualitatively different cognitive processes engaged during memory retrieval can be modulated by emotion, some of which may not require explicit awareness of the emotional nature of an item's encoding context.

One issue of interest was whether these distinct processes would engage different neural regions. Awareness of emotional stimuli has been shown to modulate processing in emotionsensitive structures, in particular the amygdala. For example, masked presentation of fear-conditioned faces elicits responses in right amygdala alone, whereas unmasked presentation results in left amygdala responses (Morris et al. 1998a). Similarly, Gläscher and Adolphs (2003) reported differential effects of left and right amygdala lesions on skin conductance responses (SCRs) to masked and unmasked emotional pictures.

In the present experiment we associated pre-experimentally neutral pictorial stimuli with positive, negative, and neutral backgrounds at study and investigated the differential neural ac- 
Table 1. Behavioral data

\begin{tabular}{lcccc}
\hline & Neutral & Negative & Positive & $\begin{array}{c}\text { Correct } \\
\text { rejection }\end{array}$ \\
\hline Hit accuracy & $0.80(0.08)$ & $0.84(0.08)$ & $0.89(0.06)$ & $0.92(0.05)$ \\
Source accuracy & $0.44(0.16)$ & $0.59(0.16)$ & $0.56(0.17)$ & $-1069(293)$ \\
RT (msec) & $964(312)$ & $977(399)$ & $953(277)$ & $1069)$ \\
\hline
\end{tabular}

Mean accuracies and reaction times (std. dev. in parentheses).

tivity elicited by these stimuli during a source memory task. We focused on the interaction between emotion effects and the accuracy of source judgements, hypothesizing that any modulatory effects of emotion on source miss trials would be consequences of modified item value, in the absence of explicit retrieval of emotional context, whereas source hit trials would reflect retrieval of item-source associations. We hypothesized that some of the emotion effects reported in previous recognition studies, specifically those in left amygdala and insula, would be dependent upon explicit retrieval of emotional contexts whereas others would reflect processing resulting from modification of the value of retrieval cues.

\section{Results}

\section{Behavior}

Recognition accuracy and associated reaction times (RTs) are shown in Table 1, together with accuracy for source judgements to old items. ANOVA of item recognition rates showed a main effect of condition $\left[F_{(1.9,30.3)}=25.5, P<0.001\right.$; degrees of freedom corrected using the Greenhouse-Geisser method]. As shown in Figure 1A, subsidiary ANOVAs revealed that items encoded in positively valenced contexts were more likely to be recognized than items encoded in neutrally $\left[F_{(1,17)}=65.3, P<0.001\right]$ or negatively $\left[F_{(1,17)}=12.5, P<0.005\right]$ valenced contexts, whereas items encoded in negative contexts were more likely to be recognized than those from neutral backgrounds $\left[F_{(1,17)}=10.7, P<0.01\right]$.

Source memory, operationalized as the proportion of correctly recognized items attracting accurate source judgments, was enhanced for stimuli associated with either negative $\left[F_{(1,17)}=12.2, P<0.005\right]$ or positive $\left[F_{(1,17)}=10.4, P<0.01\right]$ backgrounds compared to their neutral counterparts (Fig. 1B). There was no significant difference between source accuracy for positive and negative contexts $\left[F_{(1,17)}<1\right]$, and no significant differences in RTs to hits.

\section{fMRI data-Retrieval effects}

An inclusive mask incorporating the outcomes of contrasts between source hits from each of the neutral, negative, and positive conditions versus correct rejections revealed activity common to successful retrieval of emotional and neutral source memories (Table 2). Significant effects were elicited in left lateral parietal and posterior cingulate cortex, precuneus, inferior temporal lobe, and bilateral prefrontal cortex, corresponding to those reported in other event-related fMRI studies of source memory (Henson et al. 1999; Rugg et al. 2002). Effects were also seen in left anterior insula and the basal ganglia. Memory effects for source miss trials were much more restricted, being present in left cuneus and left prefrontal cortex.

\section{Emotion effects-Source hits}

Direct comparison of negative and positive source hits revealed that the former were associated with greater activity in left cerebellum, right fusiform gyrus, and right posterior parietal cortex, whereas positive hits showed greater activity in medulla oblongata. However, these effects were much more restricted than those revealed by contrasts with neutral items, so all subsequent analyses collapsed positive and negative into a single emotional condition to improve statistical power.

Contrasts between emotional and neutral source hits (Table 3 ) revealed that retrieval of emotional contexts was associated with increased activity in left amygdala. In addition there was enhanced activity in left parahippocampal gyrus and anterior insula, the brainstem, bilateral anterior cingulate cortex (ACC) subgenual cingulate, and bilateral prefrontal areas, especially left VMPFC.

\section{Emotion effects-Source misses}

Differences in activity elicited by emotional and neutral source misses are reported in Table 4 . This contrast reflects differences in neural activity arising from emotional study history in the absence of accurate recollection of that history. We observed enhanced activity for items encoded in an emotional context, compared to neutral items, in right amygdala. Differences were also evident in right occipital cortex, precuneus and lingual gyrus, bilateral fusiform areas, right inferior parietal lobule, and bilateral prefrontal areas, particularly right superior frontal gyrus.

\section{Interaction of source memory and emotion}

We next examined whether there was differential emotional activity during retrieval for source hit compared to source miss trials. In effect such a contrast reflects modulation as a function of successful episodic retrieval of emotional context (Table 5). Source hit trials were associated with greater emotional responses in left amygdala (Fig. 2). Enhanced activity was also seen in midbrain, left superior temporal gyrus, and left orbitofrontal cortex (Fig. 3).

The reverse contrast revealed greater emotion-related modulations of right amygdala activity for source miss than source hit trials (Fig. 2). Additional effects were evident in right parahippocampal cortex, cuneus, and right prefrontal cortex (Fig. 3). The regions of left and right amygdala revealed in these contrasts together with associated signal changes are shown in Figure 2.

In order to elucidate the apparent laterality of amygdala effects in source hit and source miss conditions we compared

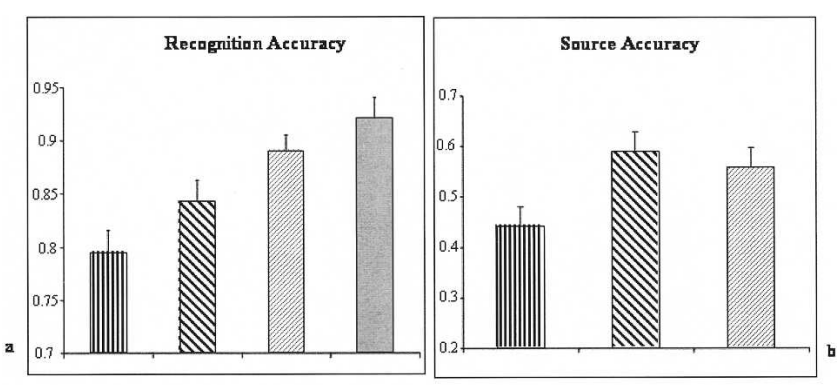

Oll Old Neutral Negative

Figure 1. Histograms showing recognition performance $(A)$ and source accuracy $(B)$, together with associated standard errors, for stimuli encoded in neutral, negative, and positive contexts. Source accuracy is conditionalized as a proportion of recognized stimuli attracting accurate source judgments. 
Table 2. Memory effects common to contrasts of neutral, negative and positive source hits with correct rejections

\begin{tabular}{|c|c|c|c|c|c|c|c|}
\hline \multicolumn{3}{|l|}{ Region } & \multirow{2}{*}{$\begin{array}{c}\text { Voxels } \\
8\end{array}$} & \multirow{2}{*}{$\frac{x}{-6}$} & \multirow{2}{*}{$\frac{y}{-72}$} & \multirow{2}{*}{$\frac{z}{45}$} & \multirow{2}{*}{$\begin{array}{c}\text { Z-score } \\
4.19\end{array}$} \\
\hline $\mathrm{L}$ & Precuneus & (BA 7) & & & & & \\
\hline $\mathrm{L}$ & Cuneus & (BA 7) & 73 & -3 & -66 & 33 & 4.22 \\
\hline $\mathrm{R}$ & Precuneus & (BA 31) & 11 & 18 & -60 & 27 & 3.93 \\
\hline $\mathrm{L}$ & Angular gyrus & (BA 39) & 5 & -45 & -66 & 33 & 3.52 \\
\hline $\mathrm{L}$ & Sup. parietal lobe & (BA 7) & 51 & -27 & -63 & 48 & 4.57 \\
\hline $\mathrm{L}$ & Post cingulate & (BA 30) & 10 & -6 & -57 & 9 & 3.76 \\
\hline $\mathrm{L}$ & Inf. temporal lobe & (BA 37) & 13 & -54 & -42 & -15 & 4.3 \\
\hline $\mathrm{R}$ & Lat. globus pallidus & & 12 & 15 & 3 & -3 & 3.41 \\
\hline $\mathrm{R}$ & Caudate body & & 5 & 12 & 6 & 12 & 3.86 \\
\hline $\mathrm{L}$ & Putamen & & 6 & -12 & 12 & -3 & 3.38 \\
\hline $\mathrm{L}$ & Insula & (BA 13) & 15 & -36 & 21 & 3 & 4.27 \\
\hline $\mathrm{R}$ & Inf. frontal gyrus & (BA 45) & 5 & 33 & 24 & 3 & 3.92 \\
\hline$L$ & Inf. frontal gyrus & (BA 46) & 59 & -51 & 45 & 0 & 4.56 \\
\hline $\mathrm{L}$ & Sup. frontal gyrus & $(\mathrm{BA} 8)$ & 16 & -3 & 33 & 45 & 5.18 \\
\hline $\mathrm{L}$ & Mid. frontal gyrus & (BA 48) & 74 & -48 & 33 & 24 & 5.06 \\
\hline & & (BA 10) & 5 & -36 & 57 & 9 & 3.9 \\
\hline
\end{tabular}

Brodmann areas (BA) are approximations based on peak coordinates.

contrast estimates from left and right amygdala in each subject. The voxels were selected using the peak coordinates from the interaction reported above, and were confirmed as lying within the amygdala of each subject on the basis of the structural images. Repeated measures ANOVA on these contrast estimates revealed a significant hemisphere by condition interaction $\left[F_{(1,17)}=27.13, P<0.001\right]$. Although choosing voxels based on the contrast between emotion effects on source hits and misses results in a partially biased measure, this test provides additional support for the lateralization of amygdala responses during retrieval of emotional memories according to the specificity of emotional information retrieved.

\section{Discussion}

The modulation of memory processing by emotion is reflected in differential neural activity during retrieval of memories formed in association with emotional contexts, and improved memory for these events. Here we describe evidence for engagement of distinct circuits during retrieval of emotional memories, demonstrating dissociable effects of emotion on the neural correlates of retrieval processing according to whether or not there is accurate recollection of the encoding context.

The behavioral findings of enhanced memory following emotional manipulation are consistent with previous work (see Christianson 1992). In particular, the dissociation between effects of positive and negative contexts on item and source memory (positive contexts enhancing memory both for context and the associated item, whereas negative contexts only enhanced memory for context without enhancing item memory) has been reported previously (Smith et al. 2004a). This dissociation may reflect distraction by the more "attention-grabbing" negative images away from associated neutral objects (impairing recognition, but not affecting the conditionalized probability of accurate source judgements) and/or different neuromodulatory effects of positive and negative emotion on memory (see Erk et al. 2003; Smith et al. 2004a). It is worth noting that accurate source judgements could be made either due to recollection of the actual context associated with each object, the context-object association formed by the subject, or an awareness of the prior emotional association without explicit recollection of the context itself. During post-scanning debriefing all subjects indicated that the great majority of source judgements followed recollection of the context and association, but it cannot be discounted that some source hit trials may have been accompanied by awareness of the valence paired with an object in the absence of contextual recollection.

One of the most notable fMRI findings was the lateralization of amygdala responses to items encoded in emotional contexts, according to whether sufficient information was retrieved to support source memory judgments. Although less well established than during encoding and consolidation of emotional memory, there is some evidence for the involvement of the amygdala at the time of retrieval. In rodents, Nader and LeDoux (1999) showed that reducing dopamine D1 receptor activation in the basolateral amygdala in a second-order fear conditioning paradigm reduces subsequent freezing responses to the second conditioned stimulus, suggesting an impairment in emotional retrieval (see also Greba and Kokkinidis 2000). Consistent with this view, Seidenbecher and colleagues (2003) showed increased synchronization of amygdalar and hippocampal theta rhythms following exposure to previously conditioned stimuli and contexts. Interactions of the amygdala with mnemonic structures during retrieval were also proposed to account for mood congruency effects in humans (Rolls and Stringer 2001).

Phelps and colleagues (1998) reported that a patient with bilateral amygdala damage showed equivalent enhancement of memory for neutral words embedded within emotional sentences to controls (see also Phelps et al. 1997; Kensinger et al. 2002 for supporting findings), suggesting that the amygdala may not be critical for emotional source memory. However, control subjects in that experiment did not report emotional arousal dur-
Table 3. Emotion effects revealed by contrasts of emotional and neutral source hits

\begin{tabular}{lllrrrrr}
\hline Region & & Voxels & $\mathbf{x}$ & $\mathbf{y}$ & $\mathbf{z}$ & Z-score \\
\hline $\mathrm{R}$ & Cerebellum & & 14 & 24 & -33 & -21 & 3.99 \\
$\mathrm{~L}$ & Parahippocampal gyrus & (BA 36) & 6 & -36 & -24 & -18 & 3.32 \\
$\mathrm{~L}$ & Sup. temporal lobe & (BA 22) & 6 & -48 & -15 & -6 & 3.11 \\
& & (BA 38) & 10 & -45 & 0 & -10 & 3.65 \\
$\mathrm{~L}$ & Amygdala & 8 & -27 & -6 & -15 & 3.24 \\
$\mathrm{~L}$ & Parahpc/lnf. frontal gyri & (BA 34/47) & 99 & -12 & -6 & -15 & 5.37 \\
$\mathrm{~L}$ & Anterior insula & (BA 13) & 5 & -42 & -3 & -6 & 4.56 \\
$\mathrm{R}$ & Subcallosal cingulate & (BA 25) & 5 & 3 & 18 & -12 & 3.93 \\
$\mathrm{~L}$ & Subcallosal cingulate & (BA 25) & 5 & -6 & 21 & -12 & 3.4 \\
$\mathrm{~L}$ & Inf. frontal gyrus & (BA 47) & 18 & -33 & 18 & -9 & 3.31 \\
$\mathrm{R}$ & Inf. frontal gyrus & (BA 47) & 5 & 33 & 27 & -6 & 4.27 \\
$\mathrm{~L} / \mathrm{R}$ & Anterior cingulate & (BA 24) & 10 & 0 & 24 & -6 & 3.73 \\
$\mathrm{R}$ & Anterior cingulate & (BA 32/24) & 8 & 3 & 39 & 3 & 4.03 \\
$\mathrm{~L}$ & Mid. frontal gyrus & (BA 11) & 5 & -30 & 39 & -12 & 4.21 \\
$\mathrm{~L}$ & Med. orbitofrontal cortex & (BA 11) & 5 & -3 & 39 & -21 & 3.62 \\
$\mathrm{~L}$ & Sup. frontal gyrus & (BA 9) & 8 & -6 & 57 & 36 & 3.61 \\
$\mathrm{~L}$ & Med. frontal gyrus & (BA 10) & 24 & -3 & 66 & 6 & 3.98 \\
\hline
\end{tabular}

Brodmann areas (BA) are approximations based on peak coordinates. 
Table 4. Emotion effects revealed by contrasts of emotional and neutral source misses

\begin{tabular}{|c|c|c|c|c|c|c|c|}
\hline Region & & & Voxels & $x$ & $y$ & $\mathbf{z}$ & Z-score \\
\hline $\mathrm{R}$ & Precuneus & (BA 19) & 18 & 18 & -78 & 39 & 3.95 \\
\hline $\mathrm{L}$ & Cuneus & (BA 18) & 30 & -6 & -75 & 15 & 4.53 \\
\hline $\mathrm{R}$ & Mid. occipital gyrus & (BA 19) & 8 & 51 & -57 & -3 & 3.77 \\
\hline $\mathrm{R}$ & Lingual gyrus & (BA 18) & 53 & 15 & -57 & 3 & 4.47 \\
\hline \multirow[t]{2}{*}{$\mathrm{L}$} & Fusiform & (BA 37) & 15 & -48 & -57 & -15 & 3.63 \\
\hline & & (BA 20) & 20 & -42 & -9 & -24 & 4.05 \\
\hline $\mathrm{R}$ & Fushiform & (BA 20) & 5 & 42 & -15 & -24 & 4.18 \\
\hline $\mathrm{R}$ & Mid. temporal gyrus & (BA 21) & 12 & 60 & -54 & 6 & 3.56 \\
\hline $\mathrm{R}$ & Inf. parietal lobule & (BA 40) & 38 & 48 & -36 & 42 & 4.61 \\
\hline $\mathrm{R}$ & Lat. globus pallidus & & 13 & 21 & -9 & 6 & 3.71 \\
\hline $\mathrm{R}$ & Amygdala & & 20 & 27 & -3 & -18 & 3.89 \\
\hline $\mathrm{R}$ & Sup. temporal gyrus & (BA 38) & 14 & 51 & 15 & -18 & 3.82 \\
\hline L & Sup. temporal gyrus & (BA 38) & 14 & -48 & 12 & -21 & 3.48 \\
\hline $\mathrm{L}$ & Mid. frontal gyrus & (BA 9) & 11 & -48 & 9 & 36 & 3.84 \\
\hline $\mathrm{R}$ & Mid. frontal gyrus & (BA 11) & 5 & 33 & 36 & -12 & 3.78 \\
\hline $\mathrm{R}$ & Inf. frontal gyrus & (BA 45) & 5 & 63 & 12 & 21 & 4.03 \\
\hline $\mathrm{L}$ & Inf. frontal gyrus & (BA 47) & 6 & -57 & 21 & 0 & 3.37 \\
\hline $\mathrm{R}$ & Sup. frontal gyrus & (BA 10) & 22 & 33 & 54 & -3 & 3.96 \\
\hline $\mathrm{L}$ & Sup. frontal gyrus & (BA 9) & 9 & -12 & 54 & 36 & 4.3 \\
\hline
\end{tabular}

Brodmann areas (BA) are approximations based on peak coordinates.

ing sentence generation, and in another experiment the same patient also showed equivalently enhanced memory for emotional words which were not associated with physiological arousal. This suggests that emotion may enhance memory through both arousal-dependent and arousal-independent mechanisms, with only the former dependent on the amygdala (see Phelps et al. 1998). It is likely that retrieval of arousing emotional sources, as in the present experiment, recruits amygdaladependent mechanisms.

Other neuroimaging studies have suggested particular roles served by the amygdala in memory retrieval. Sharot et al. (2004) reported that amygdala activity was associated with "remembered" emotional photographs, but not neutral photographs or emotional photographs which were recognized, but not recollected, in a Remember/Know (R/K) task. They suggested that activation of the amygdala was associated with the subjective feeling of arousal associated with emotional retrieval. However, as there was no overall improvement in recognition accuracy, they suggested that amygdala activity might not actually improve memory. In the present experiment we found evidence of improved source memory even in the absence of improved recognition. This suggests that amygdala activity tags a retrieved memory with a representation of its emotional valence, a crucial component of emotional memories, contributing to the subjective sense of remembering. Such a role for the amygdala is also supported by the findings of Greenberg et al. (2005), who showed a greater correlation between activity in amygdala and hippocampus during retrieval of episodic memories compared to semantic memories.

A number of previous studies have suggested lateralization of amygdala function. Morris and colleagues reported differential responsiveness of left and right amygdala respectively to "masked" and "unmasked" conditioned stimuli (Morris et al. 1998a, 1999) and to presentation of emotional faces to "blind" and "intact" visual hemifields of a patient with cortical "blindsight" (Morris et al. 2001), suggesting that left amygdala may respond only to emotional stimuli of which subjects are consciously aware, whereas right amygdala responses do not require awareness. Support for this view comes from Gläscher and Adolphs
Table 5. Areas showing interactions between emotion effects and accuracy of source memory

\begin{tabular}{|c|c|c|c|c|c|c|c|}
\hline Region & & & Voxels & $x$ & $y$ & z & Z-score \\
\hline \multicolumn{8}{|l|}{$\mathrm{SH}>\mathrm{SM}$} \\
\hline L & Sup. temporal gyrus & (BA 22) & 5 & -48 & -18 & -6 & 3.79 \\
\hline $\mathrm{L}$ & Midbrain & & 30 & -3 & -12 & -15 & 5.02 \\
\hline $\mathrm{L}$ & Amygdala & & 7 & -21 & 0 & -18 & 3.46 \\
\hline $\mathrm{L}$ & Mid. frontal gyrus & (BA 11) & 5 & -27 & 42 & -12 & 3.97 \\
\hline \multicolumn{8}{|l|}{$\mathrm{SM}>\mathrm{SH}$} \\
\hline $\mathrm{R}$ & Cuneus & (BA 18) & 12 & 6 & -75 & 18 & 4.3 \\
\hline $\mathrm{R}$ & Lat. globus pallidus & & 6 & 21 & -6 & 3 & 3.4 \\
\hline $\mathrm{R}$ & Parahip/amygdala & & 24 & 30 & 3 & -15 & 3.46 \\
\hline $\mathrm{R}$ & Sup. frontal gyrus & (BA 10) & 20 & 42 & 51 & -6 & 3.45 \\
\hline
\end{tabular}

Brodmann areas (BA) are approximations based on peak coordinates.
(2003), who investigated SCRs elicited by "masked" and "unmasked" emotional photographs in patients with damage to left, right, or bilateral amygdala. They reported reduced SCRs overall in patients with right and bilateral amygdala damage. In addition, SCRs correlated with ratings of arousal in normal subjects and right amygdala patients, but not in patients with left or bilateral amygdala damage. These findings suggest that left amygdala decodes stimulus-specific emotional information, whereas right amygdala activity is triggered automatically by arousing stimuli, without the necessity for supraliminal processing.

An alternative laterality distinction was proposed by $\mathrm{Fu}$ nayama and colleagues (2001) based upon eyeblink startle responses to emotional pictures and to "instructed fear" in patients with left and right temporal lobectomies. Damage to the right temporal lobe eliminated potentiation of startle responses to negative pictures, but not to threat trials in the instructed fear task, whereas patients with left temporal lobe damage showed normal startle responses to the emotional pictures, but not in the instructed fear task. The authors suggested that left amygdala may be specialized for verbally mediated emotional information, and right amygdala for visually conveyed emotion.

It seems parsimonious that our results reflect engagement of regions involved in the explicit processing of emotion, including left amygdala, if a recognized item elicits recollection of an emotional episode. In the absence of recollection, changes in the emotional value of an item arising from its study history, which are insufficient to allow confident source judgements, may nevertheless result in a modulation of retrieval and emotional processing. A role for left amygdala in explicit processing of emotional memories is consistent with human conditioning experiments indicating its involvement in situations where there is awareness of the contingency between an arbitrary stimulus and an unconditioned stimulus (UCS) (Morris et al. 1998a; Büchel et al. 1999; but see Büchel et al. 1998; LaBar et al. 1998 for conflicting results). Left amygdala activity has also been elicited in response to a stimulus subjects believed was associated with a risk of electric shock in an "instructed fear" paradigm (Phelps et al. 2001), and by words previously encoded in negative vs. neutral sentences (Maratos et al. 2001). 


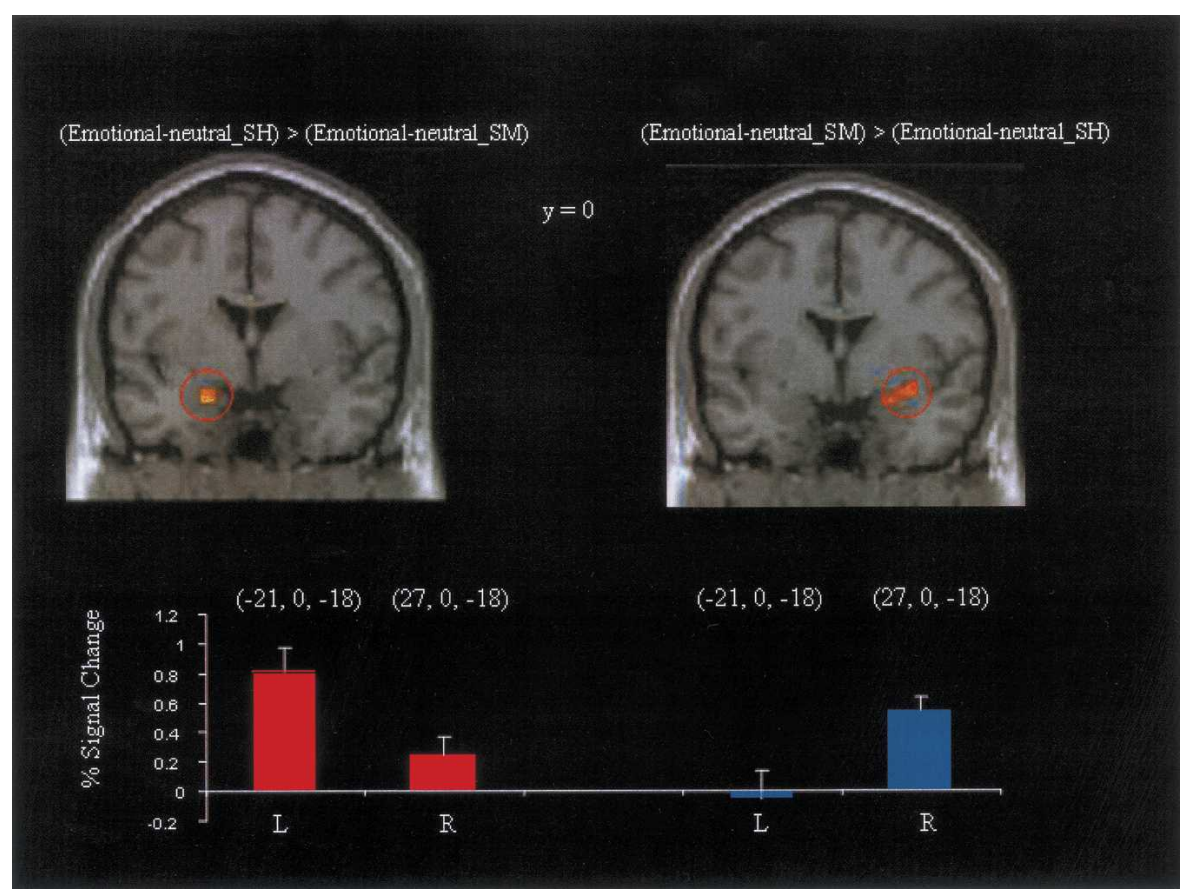

Figure 2. Areas of the left and right amygdala showing differences in emotion effects dependent on accuracy of source memory, shown at the threshold $P<0.005$ for illustrative purposes, together with $\%$ signal change (and standard errors) associated with these effects.

A number of other regions associated with emotional processing, including the ACC and OFC, were engaged by recollection of emotional contexts. The ACC is often engaged during cognitively demanding emotion processing tasks and is a candidate region for integration of input from other emotion-responsive structures, allowing appropriate guidance of behavior (Phan et al. 2002). OFC is strongly associated with representing the affective value of stimuli (see Kringelbach and Rolls 2004) and with emotion-guided decision-making (Bechara 2004). These "higherorder" emotion processing areas may be involved in accurate source discrimination based on retrieved emotional information.

Although the emotion effects described above engaged regions not normally engaged during memory retrieval, we also observed significant modulations in areas engaged by recollection of neutral memories in this and other studies. These modulations probably reflect enhancement of processes supporting visual imagery, monitoring of the products of retrieval, and guiding responses. The relative influence of emotion on emotionsensitive and mnemonic areas has been characterized (Smith et al. 2004b), showing a pattern similar to that observed for source hit trials in the present experiment.

For the source miss trials, explicit representations of emotional context or of the contingency between the object cue and the specific emotional context were not retrieved. Nonetheless, aspects of the emotional significance of cues may be indexed in the brain. In addition to effects in right amygdala, we observed activity in extra-striate visual areas and fusiform gyrus, which may reflect re-entrant processing enhancing the visual analysis of arousing stimuli (Morris et al. 1998b). Likewise, effects in lingual gyrus, an important component of the semantic memory system (Price 2000), may reflect enhanced semantic processing. Such effects may reflect different connectivity and influences of left and right amygdala. Alternatively, when emotional information is accurately recollected, attentional resources may be directed towards internal representation of the recollected context. This may detract attention from processing of external stimuli.
There are a number of outstanding issues to be addressed regarding the results of the present study. The paradigm employed required explicit encoding of emotional information in association with neutral items, and it may be that the effects of retrieving incidentally encoded emotional information may be somewhat different. D'Argembeau and Van der Linden (2004) compared the intentional and incidental encoding of emotional and neutral words, together with their contextual features. Emotionrelated differences in memory were equivalent or greater under conditions of incidental versus intentional encoding, depending on the difficulty of the contextual task. This suggests that the processes engaged in the present experiment would also be important in the retrieval of incidentally encoded information.

It is unclear whether the emotion effects observed for source hit trials depended on veridical recollection of emotional information or whether they reflect the mental representation of any kind of emotional information. In the latter case, a similar pattern of emotion effects would be expected in contrasts between false alarms attributed as being associated with emotional and neutral encoding contexts (e.g., Düzel et al. 1997). In the present experiment, there were too few false alarm trials to perform such an analysis, but it would be of interest in the future to examine false recollection effects using "lure" items, similar to items seen during encoding to increase false alarms.

One final issue is the nature of the emotion effects elicited in the absence of accurate source memories. The most likely explanation is that these effects reflect differential responses to cue items with modified emotional value as a result of their associations during encoding, though there was no direct evidence of such a change in value. However, there seem few plausible alternative explanations, and the present experiment in any case identifies clear neural indices of nonepisodic emotional retrieval processes.

In conclusion, the present findings demonstrate that retrieval activity reflecting test items' emotional study contexts can take two distinct forms, depending on the amount of information retrieved about the contexts. Episodic retrieval of an emotional episode engages a circuit incorporating left amygdala, whereas stimuli previously associated with emotional contexts which fail to elicit recollection of specific emotional information nonetheless elicit effects in right amygdala and areas processing visual and semantic aspects of the stimulus. Therefore two distinct circuits may be engaged to optimize the processing of stimuli that have acquired emotional value, and may facilitate the ability to interpret and act upon retrieved material.

\section{Materials and Methods}

\section{Subjects}

Nineteen right-handed young adults (age range 18-30 yrs, mean 21 ) were employed as subjects and remunerated at $£ 7.50 / \mathrm{hr}$. All were in good health with no history of neurological or psychiatric illness. One subject was excluded from the final analysis due to excessive movement-related artifacts. Of the remaining 18 


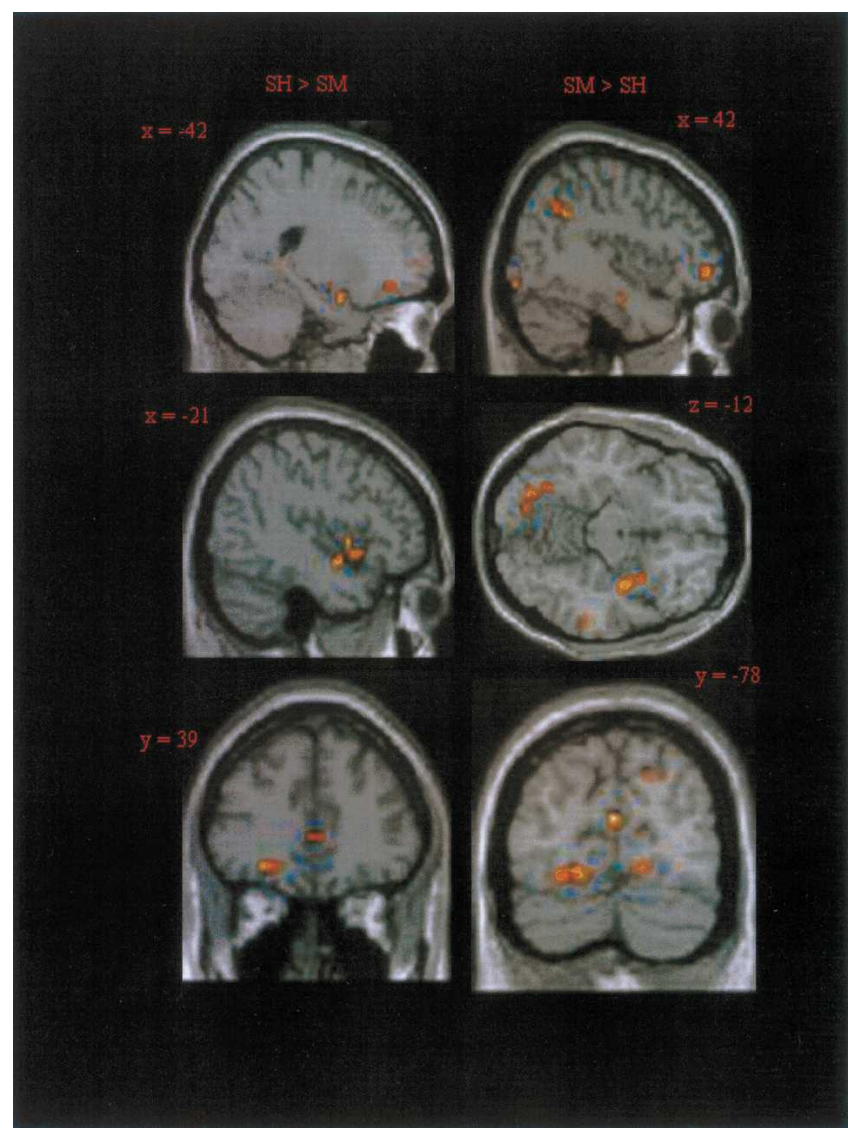

Figure 3. Representative images of areas showing interactions of emotion effects with accuracy of source memory, shown at the threshold $P<0.005$.

subjects, eight were female. The experiment was approved by the joint ethics committee of the National Hospital for Neurology and Neurosurgery and the Institute of Neurology.

\section{Stimulus materials and list construction}

Stimulus materials and lists were identical to those used in previous experiments (Smith et al. 2004a,b). In brief, stimuli consisted of two picture components, an object superimposed on a background context. Objects were presented within a yellow box to demarcate their separation from backgrounds, which were drawn principally from the IAPS (Lang et al. 1997); additional non-IAPS pictures comprised $\sim 5 \%$ of the total. A subset of this combined pool of backgrounds was selected on the basis of valence and arousal ratings (using separate five-point Likert scales) of British pilot subjects to give 180 pictures, subdivided into three sets which were either negatively, neutrally, or positively valenced. Mean (SD) valence ratings for the contexts employed were: negative $1.29(0.47)$, neutral $3.06(0.43)$, positive 4.17 (0.54). Arousal ratings were: negative $3.9(0.6)$, neutral $1.6(0.56)$, positive $3.1(0.9)$. The objects which were superimposed on these backgrounds came from a wide range of semantic categories and had also been rated during piloting to eliminate items which deviated from neutral valence or were arousing. A total of 360 objects were employed as critical stimuli.

At study, subjects were presented with one of six study lists, each consisting of 180 background/object pairs, with no more than three pictures from the same valence category presented consecutively. Each critical object was paired with a neutral background in one list, a negative background in another, and a positive background in a third. Therefore for each list 180 objects were available as new items for the associated test lists. The study phase was separated into two parts to allow rest breaks, and the first two background/object pairs in each part were neutral filler items. Each study list had three associated test lists, consisting of 180 old items, 90 new items, and four fillers. Each test list used a different set of new items, and each subject was tested on only one of the three test lists; 120 null events were randomly interspersed with the test list, allowing estimation of item-evoked responses relative to baseline. A practice study list of six background/object pairs and a practice test list of nine objects were also constructed and were used to train subjects prior to the experiment proper.

\section{Study procedure}

In the study phase, stimuli were presented via a mirror mounted on the head coil of the fMRI scanner, in direct view of the supine participant, at a distance of $\sim 50 \mathrm{~cm}$ from the projection screen. The background was initially presented alone on the screen for 3 sec. During this time subjects indicated whether they judged the backgrounds to be pleasant, unpleasant, or neutral, using a keypad in the right hand to assign them to these three categories. Three sec after presentation of the context, the critical object was superimposed centrally on the background, and subjects were required to imagine a connection between background and object. This connection was made covertly. The object and background were presented together for $4.5 \mathrm{sec}$, and the screen was then blanked for $750 \mathrm{msec}$ before presentation of the next background. Six practice trials were given prior to the study proper, during which the subjects were required to describe verbally the connections they had made between background and object, thereby ensuring they understood the task. During the study phase proper, a rest break was given after 92 trials, the first two trials of each of the subphases being neutral filler items.

\section{Test procedure}

The test phase followed the study phase after a delay of $5 \mathrm{~min}$, during which a serial subtraction task was performed to prevent rehearsal. Stimulus delivery used the same setup as during study. A white asterisk was presented against a black background for 500 msec, following which the test item was presented for $1000 \mathrm{msec}$. This was followed by a white fixation cross on a black background for $\sim 2300 \mathrm{msec}$ before presentation of the asterisk denoted the imminent onset of a new trial. This sequence of events gave a stimulus onset asynchrony (SOA) of $3.8 \mathrm{sec}$. In addition, 120 "null events," consisting of the white fixation cross for an additional $1.5 \mathrm{sec}$ in place of the white asterisk and test item, were incorporated into the test list, allowing estimation of baseline. Subjects were instructed to respond, as quickly and accurately as possible, with one of five buttons: One indicated that the object was being seen for the first time (new), another that the subject knew the object was old but could not remember the background with which it had been paired at study; and the remaining three buttons indicated that it was a remembered object from a neutral, negative, or positive background. This approach is a hybrid of those adopted in Remember/Know (R/K) and source memory experiments, and allows both validation of the accuracy of confident responses and minimizes guessing. For the purposes of analysis, trials which received a "don't know" judgment or which received an incorrect source attribution were collapsed into a single source miss category.

The test list was split into two equal parts, with the first two stimuli of each subphase being filler items. Prior to the test phase proper an example test phase was given, containing the six items from the practice study list, plus three new items. None of these items appeared in the subsequent test list. After the test phase, subjects were debriefed and questioned about perceived performance and strategies used in the task.

\section{Imaging and image processing}

MRI data were acquired from a 1.5T Siemens SONATA system equipped with a head coil. Functional images were acquired with a gradient echo-planar T2* sequence using BOLD (blood oxygenation level-dependent) contrast, with a repetition time $(\mathrm{TR})=2.7$ 
sec, giving an effective sampling rate of $\sim 2 \mathrm{~Hz}$ at both study and test. A combination of slice-tilting and z-shimming was used to minimize signal loss and distortion in basal temporal and frontal areas (Deichmann et al. 2003). Thirty-five slices of $2.5-\mathrm{mm}$ thickness were acquired, with an interslice gap of $1.3 \mathrm{~mm}$, giving whole-brain coverage, with the exception of the vertex. Data were acquired during four separate sessions (two each at study and test) with the first five volumes of each session discarded to allow for T1 equilibration effects. Images were realigned, slicetime corrected, normalized to a standard echo-planar image template, and smoothed with a Gaussian kernel with full-width half maximum of $8 \mathrm{~mm}$.

\section{Statistical analysis of images}

Data were analyzed using Statistical Parametric Mapping (SPM2; Wellcome Department of Imaging Neuroscience; Friston et al. 1995) using a random-effects analysis. Test data were modeled as 12 discrete event types: Old items from each of the three categories of old items (neutral, negative, positive) were separated according to whether they received correct source judgments (source hits), received either "old, but source unknown" or incorrect source judgments (source misses), or were incorrectly judged to be new (misses). New items were separated into those which were correctly rejected as new (correct rejections) or incorrectly judged to be old (false alarms). Categorization into valences was based on the ratings of individual subjects. A separate regressor of no interest was formed from the two filler items at the start of each list, and those trials where no response was logged. Principal contrasts were between those events which received correct responses (i.e., source hits, source misses, and correct rejections).

Regressors modeling events were convolved with a standard canonical hemodynamic response function, with movement parameters modeled as potentially confounding covariates. Linear contrasts of parameter estimates were estimated for each subject, and initial statistical parametric maps were generated. For some contrasts, positive and negative hits were collapsed to form individual "emotional source hit" and "emotional source miss" conditions, analogous to our previous ERP studies (Smith et al. 2004a). Principle contrasts were based on one-tailed $t$-tests with a significance level of $P<0.001$ (uncorrected) and a spatial extent threshold of at least five contiguous voxels $(k=5)$. Interactions were based on one-tailed tests at a more stringent threshold of $P<0.0005$, with extent threshold of five voxels. To determine generic memory effects we used "inclusive masking" to reveal voxels common to old/new contrasts across positive, neutral, and negative conditions.

\section{Acknowledgments}

This work was supported by the Wellcome Trust.

\section{References}

Bechara, A. 2004. The role of emotion in decision-making: Evidence from neurological patients with orbitofrontal damage. Brain Cogn. 55: $30-40$.

Büchel, C., Morris, J., Dolan, R.J., and Friston, K.J. 1998. Brain systems mediating aversive conditioning: An event-related fMRI study. Neuron 20: 947-957.

Büchel, C., Dolan, R.J., Armony, J.L., and Friston, K.J. 1999. Amygdala-hippocampal involvement in human aversive trace conditioning revealed through event-related fMRI. J. Neurosci. 19: 10869-10876.

Christianson, S.A. 1992. The handbook of emotion and memory. Erlbaum, Hillsdale, NJ.

D'Argembeau, A. and Van der Linden, M. 2004. Influence of affective meaning on memory for contextual information. Emotion 4: $173-188$.

Deichmann, R., Gottfried, J.A., Hutton, C., and Turner, R. 2003. Optimized EPI for fMRI studies of the orbitofrontal cortex. Neuroimage 19: 430-441.

Düzel, E., Yonelinas, A.P., Mangun, G.R., Heinze, H.J., and Tulving, E.
1997. Event-related potential correlates of two states of conscious awareness in memory. Proc. Natl. Acad. Sci. 94: 5973-5978.

Erk, S., Kiefer, M., Grothe, J., Wunderlich, A.P., Spitzer, M., and Walter, H. 2003. Emotional context modulates subsequent memory effect. Neuroimage 18: 439-447.

Friston, K.J., Frith, C.D., Frackowiak, R.S., and Turner, R. 1995. Characterizing dynamic brain responses with fMRI: A multivariate approach. Neuroimage 2: 166-172.

Funayama, E.S., Grillon, C., Davis, M., and Phelps, E.A. 2001. A double dissociation in the affective modulation of startle in humans: Effects of unilateral temporal lobectomy. J. Cogn. Neurosci. 13: 721-729.

Gläscher, J. and Adolphs, R. 2003. Processing of the arousal of subliminal and supraliminal emotional stimuli by the human amygdala. J. Neurosci. 23: 10274-10282.

Gottfried, J.A., Smith, A.P., Rugg, M.D., and Dolan, R.J. 2004. Remembrance of odors past: Human olfactory cortex in cross-modal recognition memory. Neuron 42: 687-695.

Greba, Q. and Kokkinidis, L. 2000. Peripheral and intraamygdalar administration of the dopamine D1 receptor antagonist SCH 23390 blocks fear-potentiated startle but not shock reactivity or the shock sensitization of acoustic startle. Behav. Neurosci. 114: 262-272.

Greenberg, D.L., Rice, H.J., Cooper, J.J., Cabeza, R., Rubin, D.C., and LaBar, K.S. 2005. Co-activation of the amygdala, hippocampus and inferior frontal gyrus during autobiographical memory retrieval. Neuropsychologia 43: 659-674.

Hamann, S.B. 2001. Cognitive and neural mechanisms of emotional memory. Trends Cogn. Sci. 5: 394-400.

Henson, R.N., Rugg, M.D., Shallice, T., Josephs, O., and Dolan, R.J. 1999. Recollection and familiarity in recognition memory: An event-related functional magnetic resonance imaging study. $J$. Neurosci. 19: 3962-3972.

Kensinger, E.A., Brierley, B., Medford, N., Growdon, J.H., and Corkin, S. 2002. Effects of normal aging and Alzheimer's disease on emotional memory. Emotion 2: 118-134.

Kringelbach, M.L. and Rolls, E.T. 2004. The functional neuroanatomy of the human orbitofrontal cortex: Evidence from neuroimaging and neuropsychology. Prog. Neurobiol. 72: 341-372.

LaBar, K.S., Gatenby, J.C., Gore, J.C., LeDoux, J.E., and Phelps, E.A. 1998. Human amygdala activation during conditioned fear acquisition and extinction: A mixed-trial fMRI study. Neuron 20: $937-945$.

Lang, P.J., Bradley, M.M., and Cuthbert, B.N. 1997. The International Affective Picture System(IAPS): Photographic slides. University of Florida, Gainesville, Florida.

Maratos, E.J. and Rugg, M.D. 2001. Electrophysiological correlates of the retrieval of emotional and non-emotional context. J. Cogn. Neurosci. 13: $877-891$.

Maratos, E.J., Dolan, R.J., Morris, J.S., Henson, R.N., and Rugg, M.D. 2001. Neural activity associated with episodic memory for emotional context. Neuropsychologia 39: 910-920.

Morris, J.S., Ohman, A., and Dolan, R.J. 1998a. Conscious and unconscious emotional learning in the human amygdala. Nature 393: $467-470$

Morris, J.S., Friston, K.J., Buchel, C., Frith, C.D., Young, A.W., Calder, A.J., and Dolan, R.J. 1998b. A neuromodulatory role for the human amygdala in processing emotional facial expressions. Brain 121: $47-57$.

Morris, J.S., Ohman, A., and Dolan, R.J. 1999. A subcortical pathway to the right amygdala mediating "unseen" fear. Proc. Natl. Acad. Sci. 96: $1680-1685$.

Morris, J.S., DeGelder, B., Weiskrantz, L., and Dolan, R.J. 2001. Differential extrageniculostriate and amygdala responses to presentation of emotional faces in a cortically blind field. Brain 124: $1241-1252$.

Nader, K. and LeDoux, J.E. 1999. Inhibition of the mesoamygdala dopaminergic pathway impairs the retrieval of conditioned fear associations. Behav. Neurosci. 113: 891-901.

Phan, K.L., Wager, T., Taylor, S.F., and Liberzon, I. 2002. Functional neuroanatomy of emotion: A meta-analysis of emotion activation studies in PET and fMRI. Neuroimage 16: 331-348.

Phelps, E.A., LaBar, K.S., and Spencer, D.D. 1997. Memory for emotional words following unilateral temporal lobectomy. Brain Cogn. 35: 85-109.

Phelps, E.A., LaBar, K.S., Anderson, A.K., O'Connor, K.J., Fulbright, R.K., and Spencer, D.D. 1998. Specifying the contributions of the human amygdala to emotional memory: A case study. Neurocase 4: 527-540.

Phelps, E.A., O'Connor, K.J., Gatenby, J.C., Gore, J.C., Grillon, C. and Davis, M. 2001. Activation of the left amygdala to a cognitive representation of fear. Nat. Neurosci. 4: 437-441.

Price, C.J. 2000. The anatomy of language: Contributions from functional neuroimaging. J. Anat. 197: 335-359. 
Rolls, E.T. and Stringer, S.M. 2001. A model of the interaction between mood and memory. Network 12: 89-109.

Rugg, M.D. and Allan, K. 2000. Memory retrieval: An electrophysiological perspective. In The New Cognitive Neurosciences 2nd ed. (ed. M.S. Gazzaniga), pp. 805-816. MIT Press, Cambridge, MA.

Rugg, M.D., Otten, L.J., and Henson, R.N.A. 2002. The neural basis of episodic memory: Evidence from functional neuroimaging. Philos. Trans. R Soc. Lond. B Biol. Sci. 357: 1097-1110.

Seidenbecher, T., Laxmi, T.R., Stork, O., and Pape, H.C. 2003.

Amygdalar and hippocampal $\theta$ rhythm synchronization during fear memory retrieval. Science 301: 846-850.
Sharot, T., Delgado, M.R., and Phelps, E.A. 2004. How emotion enhances the feeling of remembering. Nat. Neurosci. 7: 1376-1380.

Smith, A.P.R., Dolan, R.J., and Rugg, M.D. 2004a. Event-related potential correlates of the retrieval of emotional and non-emotional context. J. Cog. Neurosci. 15: 1-17.

Smith, A.P.R., Henson, R.N.A., Dolan, R.J., and Rugg, M.D. 2004b. fMRI correlates of the episodic retrieval of emotional contexts. Neuroimage 22: $868-887$.

Received July 22, 2004; accepted in revised form July 25, 2005. 


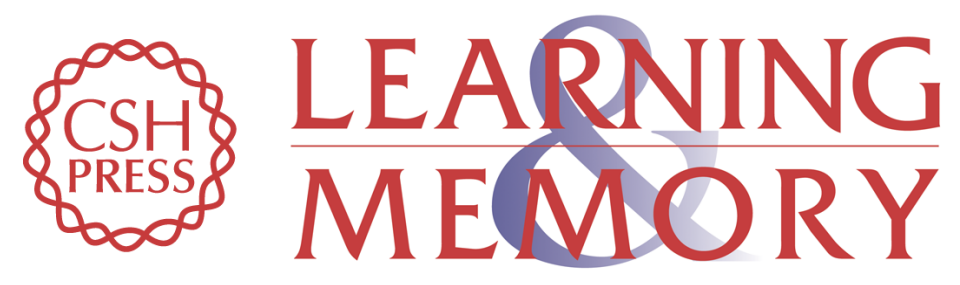

\section{Modulation of retrieval processing reflects accuracy of emotional source memory}

Adam P.R. Smith, Richard N.A. Henson, Michael D. Rugg, et al.

Learn. Mem. 2005, 12:

Access the most recent version at doi:10.1101/lm.84305

References This article cites 35 articles, 6 of which can be accessed free at:

http://learnmem.cshlp.org/content/12/5/472.full.html\#ref-list-1

License

Email Alerting Receive free email alerts when new articles cite this article - sign up in the box at the Service top right corner of the article or click here. 